\title{
UNCERTAIN GROUP DECISION-MAKING WITH INDUCED AGGREGATION OPERATORS AND EUCLIDEAN DISTANCE
}

\author{
Weihua SU, Shouzhen ZENG ${ }^{\mathrm{b}}$, Xiaojia YEc \\ ${ }^{\mathrm{a} C o l l e g e ~ o f ~ M a t h e m a t i c s ~ a n d ~ S t a t i s t i c s, ~ Z h e j i a n g ~ U n i v e r s i t y ~ o f ~ F i n a n c e ~ a n d ~ E c o n o m i c s, ~}$ \\ Hangzhou, 310018, China \\ ${ }^{\mathrm{b} C o l l e g e ~ o f ~ C o m p u t e r ~ a n d ~ I n f o r m a t i o n, ~ Z h e j i a n g ~ W a n l i ~ U n i v e r s i t y, ~ N i n g b o, ~ 315100, ~ C h i n a ~}$ \\ 'School of Mathematics and Information Science, Shanghai Lixin University of Commerce, \\ Shanghai, 201620, China
}

Received 10 November 2011; accepted 19 May 2012

\begin{abstract}
In this paper, we present the induced uncertain Euclidean ordered weighted averaging distance (IUEOWAD) operator. It is an extension of the OWA operator that uses the main characteristics of the induced OWA (IOWA), the Euclidean distance and uncertain information represented by interval numbers. The main advantage of this operator is that it is able to consider complex attitudinal characters of the decision-maker by using order-inducing variables in the aggregation of the Euclidean distance. Moreover, it is able to deal with uncertain environments where the information is very imprecise and can be assessed with interval numbers. We study some of its main properties and particular cases such as the uncertain maximum distance, the uncertain minimum distance, the uncertain normalized Euclidean distance (UNED), the uncertain weighted Euclidean distance (UWED) and the uncertain Euclidean ordered weighted averaging distance (UEOWAD) operator. We also apply this aggregation operator to a group decision-making problem regarding the selection new artillery weapons under uncertainty.
\end{abstract}

Keywords: interval numbers, Euclidean measure, IOWA operator, group decision-making.

Reference to this paper should be made as follows: Su, W.; Zeng, S.; Ye, X. 2013. Uncertain group decision-making with induced aggregation operators and Euclidean distance, Technological and Economic Development of Economy 19(3): 431-447.

JEL Classification: C44, C49, D81, D89.

Corresponding author Shouzhen Zeng

E-mail: zszzxl@163.com 


\section{Introduction}

A wide range of aggregation operators are found in the literature (Beliakov et al. 2007; Calvo et al. 2002; Torra, Narukawa 2007; Yager et al. 2011). One common aggregation method is the ordered weighted averaging (OWA) operator (Yager 1988). It provides a parameterized family of aggregation operators that include as special cases the maximum, the minimum and the average. Since its appearance, the OWA operator has been used in a wide range of applications (Chang, Wen 2010; Liu, Jin 2012; Merigó, Gil-Lafuente 2010; Wei 2012; Yager 2007; Yu 2013; Zarghami, Szidarovszky 2009; Zhang, Xu 2013; Zhou et al. 2012).

An interesting extension of the OWA operator is the induced OWA (IOWA) operator (Yager, Filev 1999). The difference is that the reordering step is no longer determined only by the values of the arguments, but could be induced by another mechanism, such as the ordered position of the arguments; in other words, the reordering can depend on the values of their associated order-inducing variables. In the last few years, the IOWA operator has received increasing attention, e.g. Chen and Zhou (2011), Liu et al. (2013), Chiclana et al. (2007), Merigó and Casanovas (2011a), Su et al. (2013), Xu and Wang (2012), Yu and Xu (2013), Wei and Zhao (2012, 2013), Yager et al. (2011).

When using the IOWA operator, it is assumed that the available information is exact numbers. However, this may not be the real situation found in the specific problem considered. Sometimes, the available information is vague or imprecise and it is not possible to analyze it with exact numbers. Therefore, it is necessary to use another approach that is able to assess the uncertainty such as the use of interval numbers (Moore 1966; Xu, Da 2002). By using interval numbers we can consider a wide range of possible results between the maximum and the minimum. In order to extend the IOWA operator to accommodate these uncertain situations, $\mathrm{Xu}$ (2006) developed the uncertain IOWA (UIOWA) operator. Basically, it is an aggregation operator that deals with uncertain information represented in the form of interval numbers. Since its introduction, several authors have developed further improvements. For example, Merigó and Casanovas (2011d) generalized it by using generalized and quasi-arithmetic means and developed the uncertain induced quasi-arithmetic OWA (Quasi-UIOWA) operator. Based on the heavy OWA (HOWA) operator, Merigó and Casanovas (2011e) developed the uncertain induced heavy OWA (UIHOWA) operator. Merigó et al. (2012) developed the uncertain induced ordered weighted averaging-weighted averaging (UIOWAWA) operator.

A further interesting extension is the one that uses OWA and IOWA operator in distance measures. Recently, motivated by the idea of the OWA operator, Xu and Chen (2008) defined the ordered weighted distance (OWD) measure whose prominent characteristic is that they can alleviate (or intensify) the influence of unduly large (or small) deviations on the aggregation results by assigning them low (or high) weights. Merigó and Gil-Lafuente (2010) developed the ordered weighted averaging distance (OWAD) operator by using the OWA operator in the Hamming distance. Zeng and Su (2011) extended the OWD and the OWAD operator to intuitionistic fuzzy sets and developed the intuitionistic fuzzy ordered weighted distance (IFOWD) operator. On the basis of the idea of the IOWA operator, Merigó and Casanovas (2011a) presented an induced ordered weighted averaging distance (IOWAD) operator that extends the OWA operator by using distance measures and a reordering of 
arguments that depends on order-inducing variables. The IOWAD generalizes the OWAD operator and provides a parameterized family of distance aggregation operators between the maximum and the minimum distance. Going a step further, Merigó and Casanovas (2011b) presented the induced Euclidean ordered weighted averaging distance (IEOWAD) operator, which uses the IOWA operator and the Euclidean distance in the same formulation. It generalizes the Euclidean ordered weighted averaging distance (EOWAD) operator and provides a parameterized family of distance aggregation operators between the maximum and the minimum distance based on a complex reordering process that reflects the complex attitudinal character of the decision-maker. The IEOWAD operator is very useful for decision-making problems because it can establish a comparison between an ideal, though unrealistic, alternative and available options in order to find the optimal choice. As such, the optimal choice is the alternative closest to the ideal one. The main advantage of the IEOWAD operator is that it is able to deal with complex attitudinal characters (or complex degrees of orness) in the decision process by using order-inducing variables. In doing so, we are able to deal with more complex problems that are closer to real-world situations. For further research on the use of the OWA and IOWA in distance measures, e.g. Karayiannis (2000), Merigó and Casanovas (2011c), Peng et al. (2013), Zeng (2013), Zeng and Su (2012).

Usually, when using the IEOWAD operator, it is assumed that the available information is clearly known and can be assessed with exact numbers. The aim of this paper is to present a new uncertain decision-making model by using the IEOWAD operator. To do so, we will develop an induced uncertain Euclidean ordered weighted averaging distance (IUEOWAD) operator, which is an extension of the IEOWAD operator with interval numbers. Therefore, it is an extension of the OWA operator that uses the main characteristics of the induced OWA (IOWA), the Euclidean distance and uncertain information represented by interval numbers. It is also a unified model that uses the uncertain IOWA (UIOWA) operator and the Euclidean distance in the same formulation. By using distance measures in the IUEOWAD operator, we can compare the real-world information with ideal information and see which alternative better fits with the interests of the decision-maker. For example, in human resource selection, we can establish an ideal candidate that would perfectly fit the company and compare it with the real-world alternatives that we have in the market and select the candidate with closest results to the ideal one. The main advantage of the IUEOWAD is that it can deal with uncertain environments represented in the form of interval numbers and with complex reordering processes of the information. In decision-making problems, this means that we are considering complex attitudinal characters of the decision-maker that reflect psychological aspects, pressure, utility, and a lot of other aspects. Furthermore, this new operator generalizes a wide range of uncertain distance measures and aggregation operators such as the uncertain maximum distance, the uncertain minimum distance, the uncertain normalized Euclidean distance (UNED), the uncertain weighted Euclidean distance (UWED) and the uncertain Euclidean ordered weighted averaging distance (UEOWAD) operator. We study some of its particular cases.

We study the applicability of the IUEOWAD operator and we see that it is very broad because all the previous studies that use the induced aggregation operators or the Euclidean distance can be revised with this new approach. For example, we can apply it to statistics, eco- 
nomics, engineering, physics and medicine. In this paper we focus on a group decision-making problem of a military unit purchasing new artillery weapons. Thus, we are able to consider a wide range of aggregation operators that could be used in the aggregation. We can see that, depending on the aggregation operator used, the results may lead to different decisions. Therefore, the decision maker is able to consider a wide range of scenarios and select the one that is in accordance with his interests. Moreover, by using several experts in the analysis, we obtain information that it is more robust because the opinion of several experts is always better than the opinion of one.

This paper is organized as follows. In Section 1 we briefly review some basic concepts. Section 2 presents the IUEOWAD operator and Section 3 analyzes different families of IUEOWAD operators. Section 4 briefly describes the decision-making process based on the developed operator and we give a numerical example in Section 5. The final section summarizes the main conclusions of the paper.

\section{Preliminaries}

In this section, we briefly review some basic concepts about the interval numbers, the IOWA and the IEOWAD operator.

\subsection{Interval numbers}

The interval number (Moore 1966; Xu, Da 2002) is a very useful and simple technique for representing the uncertainty, which has been used in an astonishingly wide range of applications. The interval numbers can be expressed in different forms. For practical reasons, we use the notation introduced by $\mathrm{Xu}$ and $\mathrm{Da}$ (2002). According to this notation, an interval number $\tilde{a}$ can be noted as: $\tilde{a}=\left[a^{L}, a^{U}\right]$, where $a^{L} \leq a^{U}$. Especially, $\tilde{a}$ is a real number, if $a^{L}=a^{U}$.

In the following, we are going to review some basic interval number operations as follows: Let $\tilde{a}=\left[a^{L}, a^{U}\right]$ and $\tilde{b}=\left[b^{L}, b^{U}\right]$ be two interval numbers, then:

1) $\tilde{a}+\tilde{b}=\left[a^{L}+b^{L}, a^{U}+b^{U}\right]$;

2) $\lambda \tilde{a}=\left[\lambda a^{L}, \lambda a^{U}\right]$, where $\lambda \geq 0$.

Different approaches have been developed for dealing with interval number such as Chen and Zhou (2011), Liu et al. (2012), Pei (2013), Xu (2013), Xu and Cai (2012), Yue (2013). In order to measure deviation between interval numbers, $\mathrm{Xu}$ (2008) introduced a distance for each pair of interval numbers as following:

Definition 1. Let $\tilde{a}=\left[a^{L}, a^{U}\right]$ and $\tilde{b}=\left[b^{L}, b^{U}\right]$ be interval numbers, then

$$
\tilde{d}(\tilde{a}, \tilde{b})=\|\tilde{a}-\tilde{b}\|=\frac{1}{2}\left(\left|a^{L}-b^{L}\right|+\left|a^{U}-b^{U}\right|\right)
$$

is called the distance between $\tilde{a}$ and $\tilde{b}$. Obviously, the smaller the value of $\tilde{d}(\tilde{a}, \tilde{b})$, the closer between $\tilde{a}$ and $\tilde{b}$. 


\subsection{The Induced OWA Operator}

The IOWA operator was introduced by Yager and Filev (1999) and it represents an extension of the OWA operator. The main difference is that the reordering step of the IOWA is carried out with order-inducing variables, rather than depending on the values of the arguments. The IOWA operator also includes the maximum, the minimum and the average operators, as special cases. It can be defined as follows:

Definition 2. An IOWA operator of dimension $n$ is a mapping IOWA: $R^{n} \times R^{n} \rightarrow R$ that has an associated weighting vector $W$ with $w_{j} \in[0,1]$ and $\sum_{j=1}^{n} w_{j}=1$ such that:

$$
\operatorname{IOWA}\left(\left\langle u_{1}, a_{1}\right\rangle, \ldots,\left\langle u_{n}, a_{n}\right\rangle\right)=\sum_{j=1}^{n} w_{j} b_{j},
$$

where $b_{j}$ is $a_{i}$ value of the IOWA pair $\left\langle u_{i}, a_{i}\right\rangle$ having the $j$ th largest $u_{i}, u_{i}$ is the order inducing variable and $a_{i}$ is the argument variable.

\subsection{The IEOWAD operator}

The IEOWAD (Merigó, Casanovas 2011b) operator is a distance measure that uses the IOWA operator in the normalization process of the Euclidean distance. Then, the reordering of the individual distances is developed with order inducing variables. For two sets $A=\left\{a_{1}, a_{2}, \ldots, a_{n}\right\}$ and $B=\left\{b_{1}, b_{2}, \ldots, b_{n}\right\}$, the IEOWAD operator can be defined as follows:

Definition 3. An IEOWAD operator of dimension $n$ is a mapping $f: R^{n} \times R^{n} \times R^{n} \rightarrow R$ that has an associated weighting vector $W$ with $w_{j} \in[0,1]$ and $\sum_{j=1}^{n} w_{j}=1$ such that:

$$
f\left(\left(u_{1}, a_{1}, b_{1}\right), \ldots,\left(u_{n}, a_{n}, b_{n}\right)\right)=\left(\sum_{j=1}^{n} w_{j} d_{j}^{2}\right)^{1 / 2}
$$

where $d_{j}$ is the $\left|a_{i}-b_{i}\right|$ value of the IEOWAD triplet $\left(u_{i}, a_{i}, b_{i}\right)$ having the $j^{\text {th }}$ largest $u_{i}$, $u_{i}$ is the order-inducing variable, $\left|a_{i}-b_{i}\right|$ is the argument variable represented in the form of an individual distances.

When using the IEOWAD operator, it is assumed that the available information is represented in the form of exact numbers. However, this may not be the real situation found in the decision-making problems. Sometimes the available information is vague or imprecise and it is not possible to analyze it with exact numbers. In this case, it is more suitable to use interval numbers to assess the uncertainty. In the following, we shall develop the uncertain induced Euclidean OWA distance (UIEOWAD). 


\section{Induced Uncertain Euclidean OWA Distance (IUEOWAD) operator}

The induced uncertain Euclidean OWA distance (IUEOWAD) operator is an extension of the IEOWAD operator that uses uncertain information in the aggregation. The main difference between the IUEOWAD and IEOWAD operators is that the IUEOWAD operator addresses uncertain information represented using interval numbers, while the IEOWAD operator uses exact numbers. The reason for using this operator is that the uncertain factors that affect our decisions are sometimes not clearly known; thus, in order to assess the problem, we must use interval numbers in order to consider the range of uncertain results that could occur in the future. By using interval numbers, we obtain a more complete aggregation operator that considers the maximum and minimum results that could occur for a given problem. Moreover, by using the IUEOWAD, we obtain a generalization that includes a wide range of uncertain distance measures and uncertain aggregation operators such as the uncertain maximum distance, the uncertain minimum distance, the uncertain normalized Euclidean distance (UNED), the uncertain weighted Euclidean distance (UWED) and the uncertain Euclidean ordered weighted averaging distance (UEOWAD) operator. It can be defined as follows.

Definition 4. An IUEOWAD operator of dimension $n$ is a mapping $f: R^{n} \times \Omega^{n} \times \Omega^{n} \rightarrow R$ that has an associated weighting vector $W$ with $w_{j} \in[0,1]$ and $\sum_{j=1}^{n} w_{j}=1$ such that:

$$
f\left(\left\langle u_{1}, \tilde{a}_{1}, \tilde{b}_{1}\right\rangle, \ldots,\left\langle u_{n}, \tilde{a}_{n}, \tilde{b}_{n}\right\rangle\right)=\left(\sum_{j=1}^{n} w_{j} \tilde{d}_{j}^{2}\right)^{1 / 2},
$$

where $\tilde{d}_{j}$ is $\left\|\tilde{a}_{i}-\tilde{b}_{i}\right\|$ value of the IUEOWAD pair $\left\langle u_{i}, \tilde{a}_{i}, \tilde{b}_{i}\right\rangle$ having the $j^{\text {th }}$ largest $u_{i}$, $u_{i}$ is the order inducing variable and $\left\|\tilde{a}_{i}-\tilde{b}_{i}\right\|$ is the argument variable represented in the form of an individual distances.

Example 1. Let $\tilde{A}=([0.1,0.5],[0.2,0.6],[0.1,0.7],[0.4,0.5])$ and $\tilde{B}=([0.2,0.8],[0.2,0.7]$, $[0.4,0.5],[0.3,0.7])$ be two set of interval numbers, then

$$
\tilde{d}\left(\tilde{a}_{1}, \tilde{b}_{1}\right)=\frac{1}{2}(|0.1-0.2|+|0.5-0.8|)=0.2 .
$$

Similarly, we have

$$
\tilde{d}\left(\tilde{a}_{2}, \tilde{b}_{2}\right)=0.05, \tilde{d}\left(\tilde{a}_{3}, \tilde{b}_{3}\right)=0.25, \tilde{d}\left(\tilde{a}_{4}, \tilde{b}_{4}\right)=0.15 .
$$

Assume the following weighting vector $W=(0.2,0.4,0.1,0.3)$ and the order-inducing variables $U=(2,3,9,6)$. If we calculate the distance between $\tilde{A}$ and $\tilde{B}$ using the UIEOWAD, then we have:

$$
f(\tilde{A}, \tilde{B})=\left(0.2 \times 0.25^{2}+0.4 \times 0.15^{2}+0.1 \times 0.05^{2}+0.3 \times 0.2^{2}\right)^{1 / 2}=0.18 .
$$

From the above example, we can see that, compared with existing kinds of uncertain induced aggregation operators (Xu 2006; Merigó, Casanovas 2011d, 2011e), the distance of two interval numbers adopted in the IUEOWAD operator can reduce computations for interval 
numbers operations. Second, final evaluation values of uncertain induced aggregation operators are interval numbers. There are many methods to compare and rank interval numbers, and different methods employed may generate different and inconsistent results. The results of our proposed operator are crisp numbers, which can avoid inconsistent results by using an interval ranking method to rank the alternatives' final evaluation values.

From a generalized perspective of the reordering step, we can distinguish between the descending IUEOWAD (DIUEOWAD) operator and the ascending IUEOWAD (AIUEOWAD) operator by using $w_{j}=w_{n-j+1}^{*}$, where $w_{j}$ is the $j$ th weight of the DUIEOWAD and $w_{n-j+1}^{*}$ the $j$ th weight of the AUIEOWAD operator.

Note that if the weighting vector is not normalized, i.e. $\sum_{j=1}^{n} w_{j} \neq 1$, then, the IUEOWAD operator can be expressed as:

$$
f\left(\left\langle u_{1}, \tilde{a}_{1}, \tilde{b}_{1}\right\rangle, \ldots,\left\langle u_{n}, \tilde{a}_{n}, \tilde{b}_{n}\right\rangle\right)=\left(\frac{1}{\sum_{j=1}^{n} w_{j}} \sum_{j=1}^{n} w_{j} \tilde{d}_{j}^{2}\right)^{1 / 2} .
$$

Similar to the IEOWAD operator, the IUEOWAD operator is also commutative, monotonic, bounded and idempotent. A further interesting issue is the problem of ties in the reordering process of the order inducing variables. In order to solve this problem, we recommend following the policy explained by Yager and Filev (1999) about replacing the tied arguments by their average. Note that in this case, it would mean that we are replacing the tied arguments by their uncertain normalized Euclidean distance.

Another interesting issue to consider is the measures for characterizing the weighting vector $W$ of the IUEOWAD operator such as the attitudinal character, the entropy of dispersion, the divergence of $W$ and the balance operator (Yager 1988; Merigó, Casanovas 2011a). The entropy of dispersion is defined as follows:

$$
H(W)=-\sum_{j=1}^{n} w_{j} \ln \left(w_{j}\right) .
$$

The balance operator can be defined as:

$$
B A L(W)=\sum_{j=1}^{n} w_{j}\left(\frac{n+1-2 j}{n-1}\right) .
$$

And the divergence of $W$ :

$$
D I V(W)=\sum_{j=1}^{n} w_{j}\left(\frac{n-j}{n-1}-\alpha(W)\right)^{2} .
$$

The degree of orness can be defined as follows:

$$
\alpha(W)=\left(\sum_{j=1}^{n} w_{j}\left(\frac{n-j}{n-1}\right)^{2}\right)^{1 / 2} .
$$




\section{Families of IUEOWAD Operators}

By choosing a different manifestation of the weighting vector in the IUEOWAD operator, we are able to obtain different types of distance aggregation operators. For example, we can obtain the uncertain maximum distance, the uncertain minimum distance, the uncertain normalized Euclidean distance (UNED), the uncertain weighted Euclidean distance (UWED) and the uncertain Euclidean ordered weighted averaging distance (UEOWAD) operator. The main advantage of using these families is that they can be very useful for the decision-maker in some specific situations. However, each family is just one particular case. Therefore, they can only be used in some particular cases, but they cannot be seen as a general model that can be used in all possible frameworks. Thus, the best way to assess all these particular cases is by using a general formulation such as the IUEOWAD operator that includes them all. Note that the particular case to be used will depend on the interests of the decision-maker in the specific problem at hand.

Remark 1. The uncertain maximum distance is obtained by setting $w_{1}=1, w_{p}=0$, for all $p \neq 1, u_{j}=\operatorname{Max}\left\{u_{i}\right\}$ and $\left\|\tilde{a}_{j}-\tilde{b}_{j}\right\|=\operatorname{Max}\left\{\left\|\tilde{a}_{i}-\tilde{b}_{i}\right\|\right\}$, and the uncertain minimum distance by setting $w_{1}=1, w_{p}=0$, for all $p \neq 1, u_{j}=\operatorname{Max}\left\{u_{i}\right\}$ and $\left\|\tilde{a}_{j}-\tilde{b}_{j}\right\|=\operatorname{Min}\left\{\left\|\tilde{a}_{i}-\tilde{b}_{i}\right\|\right\}$. More generally, if $w_{k}=1, w_{j}=0$, for all $j \neq k$, we get the Step-IUEOWAD operator. If $w_{j}=1 / n$, we get the UNED. The UWED is obtained if $u_{i}>u_{i+1}$ for all $i$. The UEOWAD operator is formed if the ordered position of $u_{i}$ is the same as the ordered position of $\tilde{d}_{j}$ such that $\tilde{d}_{j}$ is the $j$ th largest of $\left\|\tilde{a}_{i}-\tilde{b}_{i}\right\|$.

Remark 2. Other families of IUEOWAD operators can be constructed by choosing a different weighting vector. For example, when $w_{j}=1 / m$ for $k \leq j \leq k+m-1$ and $w_{j}=0$ for $j>k+m$ and $j<k$, we obtain the window-IUEOWAD operator. Note that $k$ and $m$ must be positive integers such that $k+m-1 \leq n$.

Example 2. (Window-IUEOWAD). Assume a weighting vector of dimension $7(n=7)$. If $k=2$ and $m=4$, then the weighting vector to be used in the aggregation is $W=(0,0.25,0.25,0.25,0.25,0,0)$.

Remark 3. Another particular case is the olympic-IUEOWAD. This operator is found when $w_{1}=w_{n}=0$ and for all others $w_{j}=1 /(n-2)$. Note that if $n=3$ or $n=4$, the olympicIUEOWAD is transformed in the median- UIEOWA and if $m=n-2$ and $k=2$, the windowIUEOWAD is transformed in the olympic-IUEOWAD.

Example 3. (Olympic-IUEOWAD). Assume a weighting vector of dimension $7(n=7)$. Then the weighting vector to be used in the aggregation is $W=(0,0.2,0.2,0.2,0.2,0.2,0)$.

Remark 4. Note that the generalized median can also be used as a particular case of the IUEOWAD. If $n$ is odd we assign $w_{(n+1) / 2}=1$ and $w_{j}=0$ for all others. If $n$ is even we assign for example $w_{n / 2}=w_{(n / 2)+1}=0.5$.

Example 4. (Median-IUEOWAD). Assume $n=7$. Then the weighting vector to be used is: $W=(0,0,0,1,0,0,0)$.

Remark 5. Using a similar methodology, we could develop numerous other families of IUEOWAD operators. For more information, refer to Beliakov et al. (2007), Merigó and Casanovas (2011a, 2011b), Merigó and Gil-Lafuente (2010), Xu and Chen (2008), Yager (2007), Zeng and Su (2011). 


\section{Group decision-making with the IUEOWAD operator}

In this section, we will propose a procedure for group decision-making problems with interval preference information by application of IUEOWAD operator. The main motivation for using this model is that the representation of the information is very complex and we need to use interval numbers and the opinion of several decision makers (experts) in order to correctly assess the problem. Moreover, the attitudinal character of the decision maker is very complex and we need to use order-inducing variables in order to assess it. This can be useful in a lot of situations, for example, when the board of directors of a company wants to take a decision. Obviously, the attitudinal character of the board of directors is very complex because it involves the decision of different persons and their interests may be different. Consider a group decision-making problem with uncertain preference information. Let $X=\left\{x_{1}, x_{2}, \ldots, x_{m}\right\}$ be a discrete set of alternatives, and $S=\left\{s_{1}, s_{2}, \ldots, s_{n}\right\}$ be the set of attributes. Let $E=\left\{e_{1}, e_{2}, \ldots, e_{t}\right\}$ be the set of decision makers (whose weight vector is $V=\left(v_{1}, v_{2}, \ldots, v_{t}\right), v_{k} \geq 0, \sum_{k=1} v_{k}=1$ ). Assume that $A^{(k)}=\left(\tilde{a}_{i j}^{(k)}\right)_{m \times n}$ is the interval decision matrix, where $\tilde{a}_{i j}^{(k)}=\left[\tilde{a}_{i j}^{L(k)}, \tilde{a}_{i j}^{U(k)}\right]$ is a preference value, which takes the form of interval argument, given by the decision maker $e_{k} \in E$, for the alternative $x_{i} \in X$ with respect to the attribute $s_{i} \in S$.

In general, there are benefit attributes and cost attributes in the multiple attribute decision-making problems. In order to measure all attributes in dimensionless units and facilitate inter-attribute comparisons, we introduce the following Eqs (10) and (11) (Xu 2011) to normalize each decision matrix $A^{(k)}=\left(\tilde{a}_{i j}^{(k)}\right)_{m \times n}$ to $R^{(k)}=\left(\tilde{r}_{i j}^{(k)}\right)_{m \times n}$ :

$$
\begin{gathered}
\tilde{r}_{i j}^{(k)}=\left[\tilde{r}_{i j}^{L(k)}, \tilde{r}_{i j}^{U(k)}\right]=\left[\frac{\tilde{a}_{i j}^{L(k)}}{\max _{i}\left\{\tilde{a}_{i j}^{U(k)}\right\}}, \frac{\tilde{a}_{i j}^{U(k)}}{\max _{i}\left\{\tilde{a}_{i j}^{U(k)}\right\}}\right], \text { for benefit attribute } s_{j} ; \\
\tilde{r}_{i j}^{(k)}=\left[\tilde{r}_{i j}^{L(k)}, \tilde{r}_{i j}^{U(k)}\right]=\left[\frac{\min _{i}\left\{\tilde{a}_{i j}^{L(k)}\right\}}{\tilde{a}_{i j}^{U(k)}}, \frac{\min _{i}\left\{\tilde{a}_{i j}^{L(k)}\right\}}{\tilde{a}_{i j}^{L(k)}}\right], \text { for cost attribute } s_{j} .
\end{gathered}
$$

Then the process to follow in decision making based on the UIEOWAD operator can be summarized as follows (Zeng, Su 2011):

Step 1. Normalize each decision matrix $A^{(k)}$ to $R^{(k)}$ by Eqs (10) and (11).

Step 2. Fix the ideal levels of each attribute to form the ideal strategy (see Table 1), where $I$ is the ideal strategy expressed by a fuzzy subset, $s_{i}$ is the $i$ th characteristic to consider. 
Table 1. Ideal strategy

\begin{tabular}{ccccccc}
\hline & $s_{1}$ & $s_{2}$ & $\ldots$ & $s_{i}$ & $\ldots$ & $s_{n}$ \\
\hline$I$ & $\tilde{a}_{1}$ & $\tilde{a}_{2}$ & $\ldots$ & $\tilde{a}_{i}$ & $\ldots$ & $\tilde{a}_{n}$ \\
\hline
\end{tabular}

Step 3. Calculate the order-inducing variables $\left(u_{i j}\right)_{m \times n}$ to be used in the decision matrix for each alternative $i$ and attribute $j$. Calculate also the weighting vector of the UIEOWAD operator.

Step 4. Use the uncertain weighted averaging (UWA) operator (Xu 2008) to aggregate the information of the decision-makers $E$ by using the weighting vector $V$. The result is the uncertain unified decision matrix $R=\left(\tilde{r}_{i j}\right)_{m \times n}$, where $\tilde{r}_{i j}=\sum_{k=1}^{t} v_{k} \tilde{r}_{i j}^{(k)}$.

Step 5. Calculate the distance between the ideal values and the aggregated results by using the UIEOWAD operator. Note that it is possible to consider a wide range of IUEOWAD operators, such as those described in Sections 4.

Step 6. Adopt decisions according to the results found in the previous steps. Select the alternative/s that provides the best result/s. Moreover, establish an ordering or a ranking of the alternatives from the most to the least preferred alternative to enable consideration of more than one selection.

\section{Illustrative example}

In this section, we utilize a practical group decision-making problem to illustrate the application of the developed approaches. A military unit is planning to purchase new artillery weapons and there are four feasible artillery weapons (alternatives) $x_{i}(i=1,2,3,4)$ to be selected (adapted from $\mathrm{Xu}$ 2011). When making a decision, the attributes considered are as follows: (1) $s_{1}$, assault fire capability indices $(\mathrm{m}) ;(2) s_{2}$, reaction capability indices (evaluated using 1-5 scale); (3) $s_{3}$, mobility indices (m); (4) $s_{4}$, survival ability indices (evaluated using $0-1$ scale); and (5) $s_{5}$, cost (\$). Among these five attributes, $s_{j}(j=1,2,3,4)$ are of benefit type and $s_{5}$ is of cost type. An expert group that consists of three experts $e_{k}(k=1,2,3)$ (for which the weighting vector is $V=(0.4,0.3,0.3))$ has been set up to provide assessment information on $x_{i}(i=1,2,3,4)$. These experts evaluate the alternatives $x_{i}(i=1,2,3,4)$ with respect to the attributes $s_{j}(j=1,2,3,4,5)$ and construct three uncertain decision matrices (see Tables $2-4$ ).

Table 2. Uncertain decision matrix $A^{(1)}$

\begin{tabular}{cccccc}
\hline & $s_{1}$ & $s_{2}$ & $s_{3}$ & $s_{4}$ & $s_{5}$ \\
\hline$x_{1}$ & {$[26,000,26,500]$} & {$[2,3]$} & {$[19,000,20,000]$} & {$[0.7,0.8]$} & {$[14,000,15,000]$} \\
$x_{2}$ & {$[65,000,70,000]$} & {$[3,4]$} & {$[15,000,16,000]$} & {$[0.2,0.3]$} & {$[26,000,28,000]$} \\
$x_{3}$ & {$[50,000,55,000]$} & {$[2,4]$} & {$[16,000,17,000]$} & {$[0.7,0.9]$} & {$[24,000,25,000]$} \\
$x_{4}$ & {$[40,000,45,000]$} & {$[1,2]$} & {$[26,000,28,000]$} & {$[0.5,0.6]$} & {$[14,000,16,000]$} \\
\hline
\end{tabular}


Table 3. Uncertain decision matrix $A^{(2)}$

\begin{tabular}{cccccc}
\hline & $s_{1}$ & $s_{2}$ & $s_{3}$ & $s_{4}$ & $s_{5}$ \\
\hline$x_{1}$ & {$[27,000,28,000]$} & {$[4,5]$} & {$[18,000,20,000]$} & {$[0.7,0.9]$} & {$[16,000,17,000]$} \\
$x_{2}$ & {$[60,000,70,000]$} & {$[2,4]$} & {$[16,000,18,000]$} & {$[0.3,0.5]$} & {$[26,000,27,000]$} \\
$x_{3}$ & {$[55,000,60,000]$} & {$[1,3]$} & {$[14,000,16,000]$} & {$[0.7,1.0]$} & {$[24,000,26,000]$} \\
$x_{4}$ & {$[40,000,45,000]$} & {$[2,3]$} & {$[28,000,30,000]$} & {$[0.4,0.5]$} & {$[15,000,17,000]$} \\
\hline
\end{tabular}

Table 4. Uncertain decision matrix $A^{(3)}$

\begin{tabular}{cccccc}
\hline & $s_{1}$ & $s_{2}$ & $s_{3}$ & $s_{4}$ & $s_{5}$ \\
\hline$x_{1}$ & {$[27,000,29,000]$} & {$[3,4]$} & {$[20,000,22,000]$} & {$[0.6,0.8]$} & {$[17,000,18,000]$} \\
$x_{2}$ & {$[60,000,80,000]$} & {$[4,5]$} & {$[17,000,18,000]$} & {$[0.4,0.5]$} & {$[26,000,26,500]$} \\
$x_{3}$ & {$[40,000,60,000]$} & {$[2,5]$} & {$[15,000,17,000]$} & {$[0.8,0.9]$} & {$[26,000,27,000]$} \\
$x_{4}$ & {$[50,000,55,000]$} & {$[2,3]$} & {$[29,000,30,000]$} & {$[0.4,07]$} & {$[17,000,19,000]$} \\
\hline
\end{tabular}

Since the attributes $s_{j}(j=1,2,3,4,5)$ have different dimension units, then we utilize (10) and (11) to transform the decision matrices $A^{(k)}$ into the normalized decision matrices $R^{(k)}$ (see Tables 5-7).

Table 5. Normalized uncertain decision matrix $R^{(1)}$

\begin{tabular}{cccccc}
\hline & $s_{1}$ & $s_{2}$ & $s_{3}$ & $s_{4}$ & $s_{5}$ \\
\hline$x_{1}$ & {$[0.37,0.38]$} & {$[0.50,0.75]$} & {$[0.68,0.71]$} & {$[0.78,0.89]$} & {$[0.93,1.00]$} \\
$x_{2}$ & {$[0.93,1.00]$} & {$[0.75,1.00]$} & {$[0.54,0.57]$} & {$[0.22,0.33]$} & {$[0.50,0.54]$} \\
$x_{3}$ & {$[0.71,0.79]$} & {$[0.50,1.00]$} & {$[0.57,0.61]$} & {$[0.78,1.00]$} & {$[0.56,0.58]$} \\
$x_{4}$ & {$[0.57,0.64]$} & {$[0.25,0.50]$} & {$[0.93,1.00]$} & {$[0.56,0.57]$} & {$[0.88,1.00]$} \\
\hline
\end{tabular}

Table 6. Normalized uncertain decision matrix $R^{(2)}$

\begin{tabular}{cccccc}
\hline & $s_{1}$ & $s_{2}$ & $s_{3}$ & $s_{4}$ & $s_{5}$ \\
\hline$x_{1}$ & {$[0.39,0.40]$} & {$[0.80,1.00]$} & {$[0.60,0.67]$} & {$[0.70,0.90]$} & {$[0.88,0.94]$} \\
$x_{2}$ & {$[0.88,1.00]$} & {$[0.40,0.80]$} & {$[0.57,0.60]$} & {$[0.30,0.50]$} & {$[0.56,0.58]$} \\
$x_{3}$ & {$[0.78,0.86]$} & {$[0.20,0.60]$} & {$[0.47,0.53]$} & {$[0.70,1.00]$} & {$[0.58,0.63]$} \\
$x_{4}$ & {$[0.57,0.64]$} & {$[0.40,0.60]$} & {$[0.93,1.00]$} & {$[0.40,0.50]$} & {$[0.88,1.00]$} \\
\hline
\end{tabular}


Table 7. Normalized uncertain decision matrix $R^{(3)}$

\begin{tabular}{cccccc}
\hline & $s_{1}$ & $s_{2}$ & $s_{3}$ & $s_{4}$ & $s_{5}$ \\
\hline$x_{1}$ & {$[0.34,0.36]$} & {$[0.60,0.80]$} & {$[0.67,0.73]$} & {$[0.60,0.80]$} & {$[0.94,1.00]$} \\
$x_{2}$ & {$[0.75,1.00]$} & {$[0.80,1.00]$} & {$[0.57,0.60]$} & {$[0.40,0.50]$} & {$[0.64,0.65]$} \\
$x_{3}$ & {$[0.50,0.75]$} & {$[0.40,1.00]$} & {$[0.50,0.57]$} & {$[0.80,0.90]$} & {$[0.63,0.65]$} \\
$x_{4}$ & {$[0.63,0.69]$} & {$[0.40,0.60]$} & {$[0.97,1.00]$} & {$[0.40,0.70]$} & {$[0.90,1.00]$} \\
\hline
\end{tabular}

According to the objectives of the experts, they establish a consensus ideal levels that an artillery weapon should have. The normalized results are shown in Table 8.

Table 8. Ideal artillery weapon

\begin{tabular}{cccccc}
\hline & $s_{1}$ & $s_{2}$ & $s_{3}$ & $s_{4}$ & $s_{5}$ \\
\hline$I$ & {$[0.90,1.00]$} & {$[0.85,0.95]$} & {$[0.90,1.00]$} & {$[0.90,1.00]$} & {$[0.85,0.95]$} \\
\hline
\end{tabular}

To analyze the attitudinal character of the group of experts, we consider that they use order-inducing variables shown in Table 9, which represents the complex attitudinal character in the decision process. Note that in this example, the decision-maker assumes a different attitudinal character for each alternative because the results given by each alternative are not equal. The main advantage of using order inducing variables is that we can represent complex decision processes that include psychological factors such as time pressure, personal affects to each alternative and other related aspects.

Table 9. Order-inducing variables

\begin{tabular}{cccccc}
\hline & $s_{1}$ & $s_{2}$ & $s_{3}$ & $s_{4}$ & $s_{5}$ \\
\hline$x_{1}$ & 17 & 13 & 9 & 12 & 7 \\
$x_{2}$ & 12 & 6 & 24 & 17 & 30 \\
$x_{3}$ & 16 & 14 & 12 & 10 & 8 \\
$x_{4}$ & 14 & 17 & 20 & 12 & 8 \\
\hline
\end{tabular}

With this information, we can make an aggregation to make a decision. First, we aggregate the information of the three experts to obtain a collective uncertain decision matrix. We use the uncertain weighted averaging (UWA) operator to obtain this matrix while assuming that $V=(0.2,0.5,0.3)$. The results are shown in Table 10 .

Table 10. Collective normalized uncertain decision matrix $R$

\begin{tabular}{cccccc}
\hline & $s_{1}$ & $s_{2}$ & $s_{3}$ & $s_{3}$ & $s_{4}$ \\
\hline$x_{1}$ & {$[0.37,0.38]$} & {$[0.62,0.84]$} & {$[0.65,0.70]$} & {$[0.70,0.80]$} & {$[0.92,0.98]$} \\
$x_{2}$ & {$[0.86,1.00]$} & {$[0.66,0.94]$} & {$[0.55,0.59]$} & {$[0.30,0.43]$} & {$[0.56,0.59]$} \\
$x_{3}$ & {$[0.67,0.80]$} & {$[0.38,0.88]$} & {$[0.52,0.57]$} & {$[0.76,0.97]$} & {$[0.59,0.62]$} \\
$x_{4}$ & {$[0.59,0.66]$} & {$[0.34,0.56]$} & {$[0.94,1.00]$} & {$[0.46,1.00]$} & {$[0.89,1.00]$} \\
\hline
\end{tabular}


With this information, we can use the proposed decision-making method based on the IUEOWAD operator to get the best alternative(s). In order to provide a complete analysis of the different potential results that may occur depending on the interests of the decision makers, we present a wide range of particular cases of IUEOWAD operators such as the uncertain maximum distance, the uncertain minimum distance, the UNED, the UWED, the UEOWAD, the IUEOWAD, the Step-IUEOWAD $(k=2)$ and the Olympic-UIEOWAD. Note that for the UEOWAD and the UIEOWAD operators we assume the following vector $W=(0.1,0.2,0.2,0.2,0.3)$, formed as a consensus agreement between the three experts where they evaluate the ordered argument that should have more importance in the analysis. The aggregated results obtained by using the previous particular cases of IUEOWAD operators are shown in Tables 11. The optimal choice would be the alternative closest to the ideal.

Table 11. Aggregated results

\begin{tabular}{ccccccccc}
\hline & Max & Min & UNED & UWED & UEOWAD & UIEOWAD & Step $(\mathrm{k}=2)$ & Olympic \\
\hline$x_{1}$ & 0.275 & 0.05 & 0.155 & 0.155 & 0.129 & 0.155 & 0.17 & 0.195 \\
$x_{2}$ & 0.38 & 0.02 & 0.23 & 0.252 & 0.196 & 0.208 & 0.38 & 0.229 \\
$x_{3}$ & 0.405 & 0.085 & 0.275 & 0.282 & 0.245 & 0.282 & 0.27 & 0.285 \\
$x_{4}$ & 0.45 & 0.02 & 0.268 & 0.248 & 0.227 & 0.268 & 0.45 & 0.289 \\
\hline
\end{tabular}

As we can see, for most of the cases $x_{1}$ is the optimal choice excepting for an extreme pessimistic situation where $x_{2}$ is optimal. A further interesting issue is to establish an ordering of the alternatives. This is very useful when we want to consider more than one alternative. The results are shown in Table 12.

Table 12. Ordering of the alternatives

\begin{tabular}{clcc}
\hline & Ordering & Ordering \\
\hline Max & $x_{1} \succ x_{2} \succ x_{3} \succ x_{4}$ & UEOWAD & $x_{1} \succ x_{2} \succ x_{4} \succ x_{3}$ \\
Min & $x_{2}=x_{4} \succ x_{1} \succ x_{3}$ & IUEOWAD & $x_{1} \succ x_{2} \succ x_{4} \succ x_{3}$ \\
UNED & $x_{1} \succ x_{2} \succ x_{4} \succ x_{3}$ & Step $(k=2)$ & $x_{1} \succ x_{3} \succ x_{2} \succ x_{4}$ \\
UWED & $x_{1} \succ x_{4} \succ x_{2} \succ x_{3}$ & Olympic & $x_{1} \succ x_{2} \succ x_{3} \succ x_{4}$ \\
\hline
\end{tabular}

As we can see, we get different orderings depending on the aggregation operator used. Note that the main advantage of using the IUEOWAD operator is that we can consider complex reordering processes in an uncertain framework that can be assessed with interval numbers. Another main advantage of the IUEOWAD operator is that it gives a complete view of the decision-making problem by considering different particular types of IUEOWAD operators. Thus, depending on the interests of the decision maker, he will select a different type of IUEOWAD operator that will lead him to different results and decisions. In addition, our approach is more flexible than the existing uncertain induced aggregation operators because it can provide the decision makers more choices as the ideal levels are assigned different values according to the features of the specific situations. 


\section{Conclusions}

We have introduced the IUEOWAD operator as an aggregation operator that uses the main characteristics of the IOWA operator, the Euclidean distance and uncertain information represented in the form of interval numbers. The main advantage of this operator is that it provides more complete information because it represents the information in a more complete way considering the maximum and the minimum results that can occur. Moreover, it includes many different types of uncertain distance measures and aggregation operators, such as the UNED, the UWED and the UEOWAD operator.

We have also presented an application of the new approach to a group decision-making problem concerning the selection of artillery weapons. We have seen that the IUEOWAD is very useful because it represents very well the uncertain information by using interval numbers. The main advantage of the IUEOWAD operator in decision-making is that it shows a lot of different scenarios that could happen depending on the particular type of IUEOWAD operator used in the problem. Thus, the decision maker gets a more complete view of the decision problem and is able to select the alternative that is closest to his interests. Moreover, by using order-inducing variables, it is possible to consider different scenarios according to complex attitudinal characters.

In future research we expect to develop further extensions by adding new characteristics in the problem such as the use of probabilistic aggregations. We will also consider other decision making applications such as human resource management and product management.

\section{Acknowledgments}

The work was supported by the National Funds of Social Science of China (No. 12ATJ001, 12\&ZD211), Projects in Science and Technique of Ningbo Municipal (No. 2012B82003), Ningbo Natural Science Foundation (No. 2013A610286), the MOE Project of Key Research Institute of Humanities and Social Sciences in Universities (No. 13JJD910002) and Humanities and Social Science Key Research Base (Statistics) of Zhejiang College.

\section{References}

Beliakov, G.; Pradera, A.; Calvo, T. 2007. Aggregation functions: a guide for practitioners. Berlin: SpringerVerlag.

Calvo, T.; Mayor, G.; Mesiar, R. 2002. Aggregation operators: new trends and applications. New York: Physica-Verlag. http://dx.doi.org/10.1007/978-3-7908-1787-4

Chang, K. H.; Wen, T. C. 2010. A novel efficient approach for DFMEA combining 2-tuple and the OWA operator, Expert Systems with Applications 37(3): 2362-2370.

http://dx.doi.org/10.1016/j.eswa.2009.07.026

Chen, H. Y.; Zhou, L. G. 2011. An approach to group decision making with interval fuzzy preference relations based on induced generalized continuous ordered weighted averaging operator, Expert Systems with Applications 38(10):13432-13440. http://dx.doi.org/10.1016/j.eswa.2011.04.175 
Chiclana, F.; Herrera-Viedma, E.; Herrera, F.; Alonso, S. 2007. Some induced ordered weighted averaging operators and their use for solving group decision-making problems based on fuzzy preference relations, European Journal of Operational Research 182(1): 383-399.

http://dx.doi.org/10.1016/j.ejor.2006.08.032

Karayiannis, N. 2000. Soft learning vector quantization and clustering algorithms based on ordered weighted aggregation operators, IEEE Transactions on Neural Networks 11(5): 1093-1105. http://dx.doi.org/10.1109/72.870042

Liu, H. C.; Mao, L. X.; Zhang, Z. Y.; Li, P. 2013. Induced aggregation operators in the VIKOR method and its application in material selection, Applied Mathematical Modelling 37(9): 6325-6338. http://dx.doi.org/10.1016/j.apm.2013.01.026

Liu, P. D.; Jin, F. 2012. Methods for aggregating intuitionistic uncertain linguistic variables and their application to group decision making, Information Sciences 205(1): 58-71. http://dx.doi.org/10.1016/j.ins.2012.04.014

Liu, P. D.; Zhang X.; Jin, F. 2012. A multi-attribute group decision-making method based on intervalvalued trapezoidal fuzzy numbers hybrid harmonic averaging operators, Journal of Intelligent and Fuzzy Systems 23(5): 159-168.

Merigó, J. M.; Casanovas, M. 2011a. Decision making with distance measure and induced aggregation operators, Computers \& Industrial Engineering 60(11): 66-76. http://dx.doi.org/10.1016/j.cie.2010.09.017

Merigó, J. M.; Casanovas, M. 2011b. Induced aggregation operators in the Euclidean distance and its application in financial decision-making, Expert Systems with Applications 38(6): 7603-7608. http://dx.doi.org/10.1016/j.eswa.2010.12.103

Merigó, J. M.; Casanovas, M. 2011c. A New Minkowski distance based on induced aggregation operators, International Journal of Computational Intelligence Systems 4(2):123-133. http://dx.doi.org/10.1080/18756891.2011.9727769

Merigó, J. M.; Casanovas, M. 2011d. The uncertain induced quasi-arithmetic OWA operator, International Journal of Intelligent Systems 26(1): 1-24. http://dx.doi.org/10.1002/int.20444

Merigó, J. M.; Casanovas, M. 2011e. Induced and uncertain heavy OWA operators, Computers \& Industrial Engineering 60(1): 106-116. http://dx.doi.org/10.1016/j.cie.2010.10.005

Merigó, J. M.; Gil-Lafuente, A. M. 2010. New decision-making techniques and their application in the selection of financial products, Information Sciences 180(11): 2085-2094. http://dx.doi.org/10.1016/j.ins.2010.01.028

Merigó, J. M.; Gil-Lafuente, A. M.; Martorell, O. 2012. Uncertain induced aggregation operators and its application in tourism management, Expert Systems with Applications 39(1): 869-880. http://dx.doi.org/10.1016/j.eswa.2011.07.085

Moore, R. E. 1996. Interval analysis. Englewood Cliffs, NJ: Prentice-Hall.

Pei, Z. 2013. Rational decision making models with incomplete weight information for production line assessment, Information Sciences 222(10): 696-716. http://dx.doi.org/10.1016/j.ins.2012.07.060

Peng, D. H.; Gao, C. Y.; Gao, Z. F. 2013. Generalized hesitant fuzzy synergetic weighted distance measures and their application to multiple criteria decision-making, Applied Mathematical Modelling 37(8): 5837-5850. http://dx.doi.org/10.1016/j.apm.2012.11.016

Su, W. H.; Peng, W. Z.; Zeng, S. Z.; Peng, B.; Pan, T. J. 2013. A method for fuzzy group decision making based on induced aggregation operators and Euclidean distance, International Transactions in Operational Research 20(4): 579-594. http://dx.doi.org/10.1111/itor.12015

Torra, V.; Narukawa, Y. 2007. Modelling decisions: information fusion and aggregation operators. Berlin: Springer-Verlag.

Wei, G. W. 2012. Hesitant fuzzy prioritized operators and their application to multiple attribute decision making, Knowledge-Based Systems 31: 176-182. http://dx.doi.org/10.1016/j.knosys.2012.03.011 
Wei, G. W.; Zhao, X. F. 2012. Some induced correlated aggregating operators with intuitionistic fuzzy information and their application to multiple attribute group decision making, Expert Systems with Applications 39(2): 2026-2034. http://dx.doi.org/10.1016/j.eswa.2011.08.031

Wei, G. W.; Zhao, X. F. 2013. Induced hesitant interval-valued fuzzy Einstein aggregation operators and their application to multiple attribute decision making, Journal of Intelligent and Fuzzy Systems 24(4): 789-803.

Xu, Y. J.; Wang, H. M. 2012. The induced generalized aggregation operators for intuitionistic fuzzy sets and their application in group decision making, Applied Soft Computing 12(3): 1168-1179. http://dx.doi.org/10.1016/j.asoc.2011.11.003

$\mathrm{Xu}, \mathrm{Z}$. S. 2006. Induced uncertain linguistic OWA operators applied to group decision making, Information Fusion 7(2): 231-238. http://dx.doi.org/10.1016/j.inffus.2004.06.005

$\mathrm{Xu}, \mathrm{Z}$. S. 2011. Attribute weights determination models for consensus maximization in multiple attribute group decision-making, International Journal of General Systems 40(7): 755-774. http://dx.doi.org/10.1080/03081079.2011.594798

Xu, Z. S. 2013. Group decision making model and approach based on interval preference orderings, Computers \& Industrial Engineering 64(3): 797-803. http://dx.doi.org/10.1016/j.cie.2012.12.013

Xu, Z. S.; Cai, X. Q. 2012. Uncertain power average operators for aggregating interval fuzzy preference relations, Group Decision and Negotiation 21(3): 381-397. http://dx.doi.org/10.1007/s10726-010-9213-7

$\mathrm{Xu}, \mathrm{Z}$. S.; Chen, J. 2008. Ordered weighted distance measure, Journal of Systems Science and Systems Engineering 17(4): 432-445. http://dx.doi.org/10.1007/s11518-008-5084-8

Xu, Z. S.; Da, Q. L. 2002. The uncertain OWA operator, International Journal of Intelligent Systems 17(6): 569-575. http://dx.doi.org/10.1002/int.10038

Yager, R. R. 1988. On ordered weighted averaging aggregation operators in multi-criteria decision making, IEEE Transactions on Systems, Man and Cybernetics B 18(1):183-190. http://dx.doi.org/10.1109/21.87068

Yager, R. R. 2007. Centered OWA operators, Soft Computing 11(7): 631-639. http://dx.doi.org/10.1007/s00500-006-0125-z

Yager, R. R.; Filev, D. P. 1999. Induced ordered weighted averaging operators, IEEE Transactions on Systems, Man and Cybernetics B 29(1): 141-150. http://dx.doi.org/10.1109/3477.752789

Yager, R. R.; Kacprzyk, J.; Beliakov, G. 2011. Recent developments on the ordered weighted averaging operators: theory and practice. Berlin: Springer-Verlag. http://dx.doi.org/10.1007/978-3-642-17910-5

Yu, D. J. 2013. Intuitionistic fuzzy geometric Heronian mean aggregation operators, Applied Soft Computing 13(2): 1235-1246. http://dx.doi.org/10.1016/j.asoc.2012.09.021

Yu, X. H.; Xu, Z. S. 2013. Prioritized intuitionistic fuzzy aggregation operators, Information Fusion 14(1): 108-116. http://dx.doi.org/10.1016/j.inffus.2012.01.011

Yue, Z. L. 2013. Group decision making with multi-attribute interval data, Information Fusion 14(4): 551-561. http://dx.doi.org/10.1016/j.inffus.2013.01.003

Zarghami, M.; Szidarovszky, F. 2009. Revising the OWA operator for fuzzy stochastic multi criteria decision making, European Journal of Operational Research 198(1): 259-265.

http://dx.doi.org/10.1016/j.ejor.2008.09.014

Zeng, S. Z. 2013. Some intuitionistic fuzzy weighted distance measures and their application to group decision making, Group Decision and Negotiation 22(2): 281-298. http://dx.doi.org/10.1007/s10726-011-9262-6

Zeng, S. Z; Su, W. H. 2011. Intuitionistic fuzzy ordered weighted distance operator, Knowledge-based Systems 24(8):1224-1232. http://dx.doi.org/10.1016/j.knosys.2011.05.013 
Zeng, S. Z; Su, W. H. 2012. Linguistic induced generalized aggregation distance operators and their application to decision making, Economic Computation and Economic Cybernetics Studies and Research 46(2): 155-172.

Zhang, Z.; Xu, Z. S. 2013. On continuity of ordered aggregation operators, International Journal of Intelligent Systems 28(4): 307-318. http://dx.doi.org/10.1002/int.21577

Zhou, L. G.; Chen, H. Y.; Liu, J. B. 2012. Generalized power aggregation operators and their applications in group decision making, Computers \& Industrial Engineering 62(4): 989-999.

http://dx.doi.org/10.1016/j.cie.2011.12.025

Weihua SU has an MSc and a PhD degree in statistics from Xiamen University, China. He is a Professor in College of Mathematics and Statistics, Zhejiang University of Finance and Economics. He has published more than 90 papers in journals, books and conference proceedings including journals such as Statistics Research, Economic Research, Knowledge-based Systems and Group decision and Negotiation. He has published 3 books. He has participated in several scientific committees and serves as a reviewer in a wide range of journals including Computers \& Industrial Engineering, International Journal of Information Technology and Decision Making, Information Sciences and European Journal of Operational Research. His current research interests are aggregation operators, decision making and comprehensive evaluation.

Shouzhen ZENG graduated from the Tianjin University and obtained the Master degree in applied mathematics. At present, he is studying his in-service doctor of applied statistics in Zhejiang Gongshang University. Currently he is a full-time Lecture in Zhejiang Wanli University.

He has published more than 40 papers in journals, books and conference proceedings including journals such as Statistics Research, Economic Research, Knowledge-based Systems and Group Decision and Negotiation. His main research interests are aggregation operators, decision making, comprehensive evaluation and uncertainty.

Xiaojia YE graduated from the Zhejiang Gongshang University and obtained her Master's degree in Statistics. At present, she is studying her in-service doctor of applied statistics in Zhejiang Gongshang University. Her main research interests are aggregation operators, decision making, comprehensive evaluation and uncertainty. 